\title{
Development of Software Tool Support for Enterprise Architecture in Small and Medium-Sized Enterprises
}

\author{
Joost Dumeez, Maxime Bernaert, and Geert Poels \\ Department of Management Information Systems and Operations Management \\ Faculty of Economics and Business Administration, \\ Ghent University, Tweekerkenstraat 2, B-9000 Ghent, Belgium \\ \{Joost. Dumeez, Maxime. Bernaert, Geert. Poels\} @UGent. be
}

\begin{abstract}
Throughout recent years a lot of research has been done to develop enterprise architecture (EA) approaches for large and complex enterprises. Consequently, an array of tools has been developed for these large enterprises to aid in EA management. However, traditional small and medium-sized enterprises (SMEs), which are very important for economy, have to a great extent been neglected. Recently research has been done towards a new EA approach for SMEs. The approach is called CHOOSE. As tool support is almost indispensable in complex environments, the need for tool support was quickly experienced while doing case studies in SMEs. Unfortunately, tool support is already rated low on usability by EA practitioners in large companies. A different approach was required to provide tool support for managers in SMEs. The developed software tool already received positive feedback from managers.
\end{abstract}

Keywords: Enterprise architecture, software tool support, small and mediumsized enterprise, CHOOSE.

\section{Introduction}

A common analogy is often made between enterprise architecture (EA) and architecture in construction. When an architect is asked to design a house, the focus will be on where the staircase has to be or how many rooms or balconies there have to be. The specifics of the color or brand of paint are not of interest yet. The future habitant agrees on some kind of blueprint, a master plan that will serve as the starting point for more detailed decisions. This high level blueprint will show the major functions of the house and how these have to be constructed. As future occupant of the house this high level and abstract representation is probably the most informative. On the other hand, the engineer or constructor is probably more interested in a detailed elaboration.

When companies became bigger and more complex the need for EA arose. The advantages of EA for large enterprises have been widely acknowledged in practice and literature [1]. Landenberg and Wegmann [2] investigated which aspects of EA were being researched. One of their conclusions was that interest in the field is currently growing, given the increase in publications. Furthermore, Ernst et al. [3] found in their survey that in practice, EA is also of growing importance. However, most of this research is targeted towards architecture in large enterprises. But while small and medium-sized enterprises (SMEs) are considered the backbone of our 
economy [4], little research has been done towards EA approaches for those SMEs. In a literature research concerning information systems in SMEs [5], not one paper was found from 1979 to 2008 about EA in SMEs.

In response to this lack of research about EA for SMEs, Bernaert and Poels proposed a new EA approach for SMEs [6;7]. A metamodel was created, focusing on the essential dimensions and characteristics of EA in the context of SMEs. The metamodel was called the CHOOSE metamodel, which is an acronym for "keep Control, by means of a Holistic Overview, based on Objectives and kept Simple, of your Enterprise".

Just as tool support is widely considered indispensable in large companies, the need for it was also quickly identified in SMEs by several case studies. However, as existing tools are already considered low on usability by EA practitioners, a different approach is proposed in this research: a software application for tablets. As SMEs usually rely on insight from the CEO and do not have any enterprise architects on staff, this software application had to be useable for management.

In the next chapter, the problem statement and goal of this research are discussed. In chapter three, existing tool support is first considered. In the fourth chapter, our solution towards a user-friendly tool for the EA approach CHOOSE is then proposed. The last chapter concludes with a summary and future research directions.

\section{Problem Statement and Goal of the Research}

\subsection{Specific Research Problem}

While developing the EA approach CHOOSE, case studies were performed in SMEs. Even after the first tests, it was clear that tool support was indispensable. Working on a whiteboard with post-its was not practicable (Fig. 1).

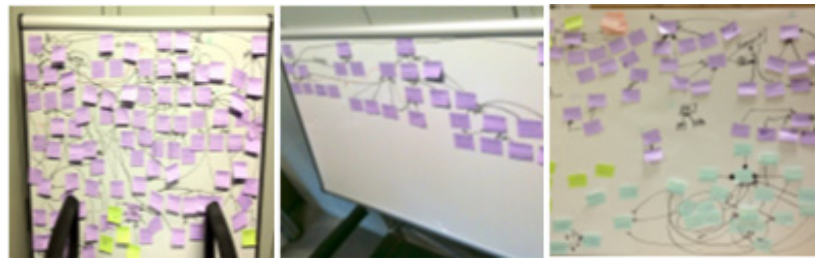

Fig. 1. Need for tool support during case studies

First of all, input was very slow, having to write every element on a separate postit. If a goal had to be corrected, a new post-it had to be made. Once the model was finished, it was impractical to store away three whiteboards and present it on a meeting the week after. More importantly, finding an element could take a while and making any sort of analysis was out of the question. Academic sources also widely support the necessity of software tool support.

"For EAs to be useful and provide business value, their development, maintenance, and implementation should be managed effectively and supported by tools." [8]

"EA management should be supported by tools, which support distributed access to consistent data, offer the possibility to structure the information managed, and also aid users in filling out their role in the EA management process." [3] 


\section{Related Research}

\section{1 $\quad$ Existing Tool Support}

Kurpjuweit and Winter [9] identify three different objectives for which tools can be used. They can be used to document and communicate, they can be used to analyze and explain or they can be used to design the EA. Typically after selecting an EA approach and selecting the preferred modeling language, there are software tools available to model your enterprise. Several options are available on the market, for example IBM's system architect [10] or Metis from Troux technologies [11]. However most of these tools are focused on the design aspect and are solely used to model the enterprise.

Based on a survey performed by the university of Munich, Ernst et al. summarize how different tools perform according to different criteria and different scenarios [3]. They came up with a kiviat diagram, showing the minimum, maximum, and median score obtained by the different tools (Fig. 2). We can see that even for EA practitioners, the average usability of the different tools scores rather low. One reason was that all tools came with predefined metamodels. Some came with up to 400 entities with corresponding associations. Even for daily practitioners this is perceived to be very complex, making the tools hard to work with in practice. Especially the visualization needed improvement. Two major issues occur when visualizing an EA with existing tools. First, the automated generation of adequate visualization is mostly not possible. The second issue is that the semantics of those visualizations are often not properly defined. This leaves a lot of room for interpretation. These issues are both explained by the simple fact that most tools are drawing tools where visualizations are manually created. This often causes practitioners to use certain symbols or links out of context. Although the model is understandable for the creators of the model, the tool can no longer interpret the results correctly. The result is a drawing rather than a model. A nice quote from this paper that summarizes this paragraph: "drawing is no management".

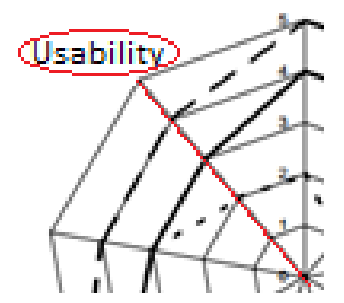

Fig. 2. Diagram showing a low usability rating

The graphical user interface of a typical EA tool is usually composed of three different components (Fig. 3 from left to right). The content explorer, a canvas to do the modeling itself, and a concept explorer with the different modeling language constructs. Often this works by dragging and dropping the different constructs from the concept explorer to the canvas and thus slowly building up the different models. 


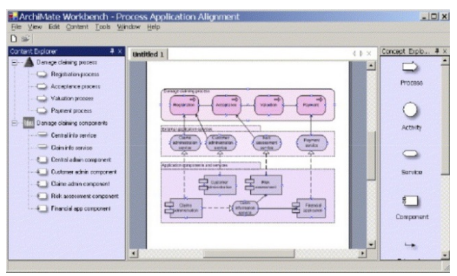

Fig. 3. Typical EA tool

\section{Solution Approach}

While it has been made clear that tool support is needed, one of the key words in the abbreviation CHOOSE was 'Simple'. Given the resource poverty of SMEs [12], they are unable to staff an enterprise architect, or pay expensive consulting fees. As described above, modeling tools are typically aimed towards enterprise architects. So while developing a tool for this new approach, it was clear that contrary to the above tools, a different approach would be required for SMEs. Even practitioners in big companies rated the existing tools rather poor in usability. Furthermore these tools expect the users to be fully aware of the models and modeling languages.

To find an approach towards tool development, a methodology that is aimed to achieve user-friendly interfaces was adopted from Cooper et al [13]. He was one of the first to criticize traditional software development processes and was the first to pioneer a software development process that was based on the users' needs. How could a good product be created when it did not take into account the users' goals, needs and motivations? The improved software development process that he proposed now emphasizes the design aspect (Fig. 4).

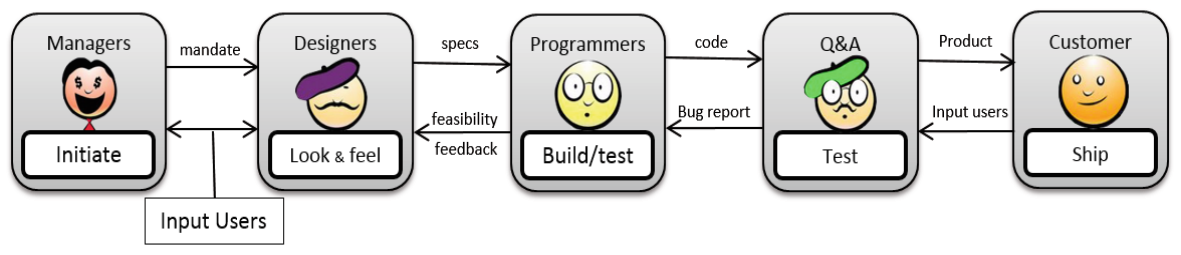

Fig. 4. Software development process

The first step, called 'initiate', is the step where the need for a certain program is identified. The need for tool support has already been discussed as being necessary to support an EA approach. The necessity of this new EA approach has also been shortly dealt with in the introduction. As the tool is currently still under development (build phase), the last two steps in the process will not be dealt with in this paper. The technicalities of the building itself are also beyond the scope of this paper. In the next section we will thus focus on the design step. An approach towards design is introduced in the next section. 


\subsection{Goal-Directed Design}

The task of the design step in Fig. 4 should not only be about the appearance of a product. When properly deployed it should identify user requirements and take into account his/her behavior. Design should be about product definition, based on the goals of users, needs of the business and the constraints of technology. Design is defined in a broader sense.

Just as we made an analogy with architecture when arguing that EA is needed, a similar reasoning can be made here. In the case of an architect, he/she will have to understand how the people live and work before he/she can start designing a house. $\mathrm{He} / \mathrm{she}$ then should sketch the spaces to support and facilitate those behaviors. For the architect, designing a house is more than making the house look pretty.

This broader approach towards design is called 'interaction design'. Interaction design is more than making sure something looks pretty; interaction design is about understanding the users and knowing cognitive principles.

An approach to implement this design philosophy is proposed by Cooper et al. [13]. In short, this approach is called 'goal-directed design'. The main steps followed in a goal-directed design approach are shown in Fig. 5.

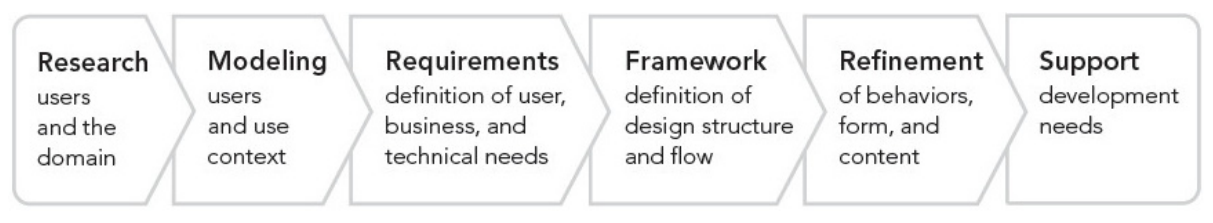

Fig. 5. Goal-directed design process

Research. The research phase consists mainly of understanding the future user and knowing who that future user will be. In the research done to develop the CHOOSE approach it was already clear that the CEO would have to be closely involved. There are two reasons illustrating the need for the CEO's involvement.

Firstly in SMEs, employees generally do not know the structure, let alone know why they are doing something. It is generally only the entrepreneur who knows the whole working of the company and this distinguishes SMEs from larger companies, because a CEO can have an overview of its SME. Secondly, in SMEs, the job description for employees is often vaguely defined and an overview of tasks or responsibilities is often missing [14].

In a study performed by Hankinson et al. [15], managers responded that they worked between 50 and 60 hours a week. Most time was spent in meetings and informal interactions $(50 \%)$ and on the phone $(40 \%)$. The remainder was spent travelling. Only 5 hours or less than $10 \%$ of time was used on strategic issues, personal analysis, and analyzing results. Notably, managers generally indicated that their time management was poor. To get a better insight in the lifestyle of the manager, a small amount of managers was asked to keep a very detailed schedule of their workweek. In this small-scale test, the results, stated above, could be verified. We clearly saw that managers were very busy with day-to-day activities. This left little time in between for working on strategic issues [16]. 
Another important factor to keep in mind is that managers are not expert technology users. According to [17], users can be divided in three types: experts, willing adopters and mainstreamers. Mainstreamers are people who do not use technology to use the technology. They use it to get something done. They use just a few key features and do not care about any other.

Modeling. In the modeling step, a persona is defined, which is the portrait of what the ideal user would be. Additionally a list of goals of what the user is interested in is composed. This is usually input from the research step. By completely defining a 'persona', programmers and designers always keep focus on who they are making the program for. Usually, programmers talk about 'the end user'. By making the end user an actual person, it restricts them of stretching the 'end user' to fit the situation. 'This is easy' might be true for the programmers, but may not be the case for the defined persona, and obliges programmers to rethink their approach to fit the persona's needs.

Because a complete persona definition is beyond the scope of this paper, a small summary is offered. Our target user is a manager in an SME, who is generally not technology savvy. He/she often does not know about the specifics of EA. Though, if he/she would use an EA approach, he/she would want it to be simple while still offering value. It would have to aid in keeping an oversight of the company.

Requirements Definition. While developing the CHOOSE approach, a list of eleven main criteria were found for EA in an SME context [7]. As the tool is used to support this approach it should at least be able to achieve these criteria. These criteria will serve as a guideline when setting requirements for the tool.

Requirements for tools can be thought of as consisting of objects, actions, and contexts. For example, for a calling application this might be something like: 'Call (action) a person (objects) directly from an appointment (context)'. As it can be quite difficult to extract information in this format, it is easier to separate them into data, functional and contextual requirements [18].

Data Requirements. These requirements are the objects and information that our users want from our tool. As can be identified by the criteria from EA, we need data output that can give an overview of the company. Furthermore a description of how things are done in the company is needed. A focused view will provide a more detailed view. This output furthermore has to be usable in other often-used programs like Excel and PowerPoint.

Functional Requirements. A mobile application was chosen to fit the busy schedule of a manager. Since there is little time to work on strategic matters during the day, the only adoption of an application would happen if it could be used during downtime. It has to be useable when they have a few minutes spare time in a waiting room or maybe five minutes before they go to bed. Showcased by the popularity of the iPad and other Android tablets, tablets are on the rise. Even in SMEs, it is becoming the standard tool that every manager has with him/her [19]. A tablet was chosen over a mobile phone because it offers almost the same mobility while giving more screen estate. 


\section{Contextual Requirements}

- $\quad$ Business Requirements: It has to be cheap to implement [12].

- Experience Requirements: If we want managers to spend their precious time on EA and use this tool, it has to be appealing and fun.

- Technical Requirements: It has to work on a platform that can complement a manager his/her usage pattern. As the CHOOSE metamodel is still being refined, the software architecture should be flexible to allow changes to be adapted quickly.

Summary of Tool Criteria. The following list of five criteria is proposed for this tool. These will be used as guidance when developing the application:

1. Offer a focus view of enterprise components

2. Offer a holistic overview of the firm

3. Simplicity, the tool has to be easy to use

4. Fit in day-to-day activities of manager

5. Fun and appealing

Design Framework. Considering usability was an issue with existing tools for EA practitioners, making the tool usable for managers would be challenging. If we go back to the roots of usability, Vitruvius is considered as being the first student of ergonomics and usability [17]. His questions were based around three key words:

- Firmitas: How durable is the design and does it have strength?

- Utilitas: How useful is the design and does it fit the user's needs?

- Venustas: How beautiful is the design?

What Vitruvius did not suggest was how to achieve these objectives. However, directions to achieve these objectives are offered by Davidson et al. [20]. They found an important link between mental models and usability.

Norman [21], an academic researcher in interface design, argues that design is often constrained by culture. People want to be able to foresee what an object will do. When an interaction with a system is deeply engrained through cultural learning it is hard to change the way people interact with an object. The easiest way to make an interface design consistent with how a user expects it to behave is by mimicking the physical product. For example, calculator applications or calendar applications often mimic the behavior of the physical products. Unfortunately, as is the case with our application, there is not always a physical object that can be mimicked. The insight to decrease the discrepancy between how an object behaves and how a user expects it to behave is offered by Norman [22], Cooper et al. [13] and IBM [23]. They have discerned three models of a system:

- Mental Model: How a user perceives that a product works.

- Implementation Model: Actual way the system works internally (programmer's perspective).

- Represented Model: The way the application is represented to the user.

According to Davidson et al. [20], who researched this link between usability and mental models, the program will be perceived easier to use and easier to understand the closer the Represented Model resembles the Mental Model. 
Several design methods are used to support and influence these mental models of the user: simplicity, familiarity, availability, flexibility, feedback, safety, and affordance are proposed by IBM [24]. By following these methods, the user's mental model will be better aligned with the represented model. According to [20], the application will then have higher usability.

Simplicity has been one of the key words throughout the development of the CHOOSE approach (the S stands for Simple). Additionally it came out as one of the main criteria during the requirements definition. As simplicity is the main guideline, in the next section this is the only design method that is further zoomed into. A design framework is furthermore also concerned with detailed design to make the application look beautiful. Although beauty is subjective, a lot of guidelines were taken into account, though are not further elaborated here and will be presented in a thesis.

Simplicity: Less Is More. When visiting a website it can sometimes feel like walking through Times Square: You see a myriad of different advertisement billboards each with catchy slogans like "The next big thing", "Probably the best beer in the world", "The real thing". Many of these billboards are very flashy with vibrant colors and flashing lights, trying to get the passer-by its attention. Every brand competes with each other to make the billboard that pops out the most, trying to capture the so important passer-by glance.

However, people are impatient and they can only process a limited amount of information [24]. The more content you show, the smaller your chances will be of them to notice your most important content. Less really is more in this case.

Even small details like words, colors, buttons can add to the already heavy load a person has to process. Removing irrelevant options, slogans, and content decreases this load on users. Designing a user interface in a clever way can further diminish this load and enhance user experience [17].

Thompson et al. [25] did an experiment to find out whether users preferred features over usability or vice versa. They concluded that features sell a product better, but when users were able to test the product before their purchase they would buy the product with better usability over the product with more features. In software, the cost of adding extra features is close to nothing. Considering people appear to buy products based on features, this leads to features piled upon features. The problem is that with each extra feature that is added, the product becomes more difficult to use.

Iyengar and Lepper [26] set up multiple experiments to test whether people like more or less choice. All results obtained showed significant higher sales revenues when offering only a handful of options. They also found that people where more satisfied with what they bought when being offered fewer options. Because it offers a person a sense of control, people prefer to have a choice over no choice at all. However if that choice exceeds a handful of options, choice can get overwhelming.

\subsection{Tool Presentation}

During the requirements definition stage in the goal-directed design process, five criteria were derived for our tool (Section 4.1.3). Furthermore simplicity, familiarity, 
availability, flexibility, feedback, safety, and affordance were the design methods that were introduced in the design framework (Section 4.1.4). While presenting the tool, some criteria and design methods will be referred to where they are applicable.

One aspect of the CHOOSE approach is to build up a goal tree. To showcase the tool, an extract from a case study is presented. The case study was performed in an SME that sells tires and does some maintenance on cars. One of the goals of this company was to increase its customer base. Two possible scenarios were considered. The first scenario was opening a new shop, the second was to increase visibility through improved signing of the building and better online visibility. To showcase the tool, the goal 'online visibility' is added to the goal tree (Fig. 6).

When starting up the application, the user is presented with a playful start screen. At first glance the start screen is already radically different from traditional approaches. Firstly it has to entice the manager of working with the program when he/she has some spare time (Criterion 5). Another important criterion is that no outside help is needed to exercise EA (SMEs' resource constraint). At the bottom right a question mark is shown. Whenever in doubt about some concept, the manager can quickly get an explanation (Design method: safety). In our case we head over to the goals part of the application and start the wizard for adding a new goal. In step 1 the name of the goal and a description can be added. In the following screen, since 'online visibility' is one of the goals to achieve a general increase in visibility, the latter goal is selected as the upper goal. Next we want this goal to be assigned to the marketing expert. This is done by browsing through the actor tree and selecting our marketing expert. Next we need a process to operationalize this goal. Here 'manage social media' is selected to operationalize 'online visibility'. Finally 'Facebook' is selected to be the online platform of choice. Facebook will serve as input and output for our operation 'manage social media' and is thus also of concern for our goal 'online visibility'. Finally, in the last step, a small summary is shown before the goal can be saved. When we return to the 'goals' part of the application, we can now browse to the goal 'online visibility' (Fig. 7). In this view, the file explorer from Microsoft Windows is mimicked which should make it very intuitive for users to browse through the goals tree (Design method: familiarity). At the right hand side a focused view of all the connected elements is offered (Criterion 1). Note that very few buttons are offered, providing just enough features (Criterion 3). The main button here is the add button at the top right corner. This started the wizard that guided us through the procedure of adding our goal 'online visibility'. By guiding the user step by step, information is shown in chewable chunks, so it never becomes overwhelming for the user (Criterion 3). Every model can be searched through in the same way, offering a consistent user experience (Criterion 3).

(Prototype: https://www.dropbox.com/sh/03vyn790ilwggsv/G_uDPwJy00/CHOOSE) 


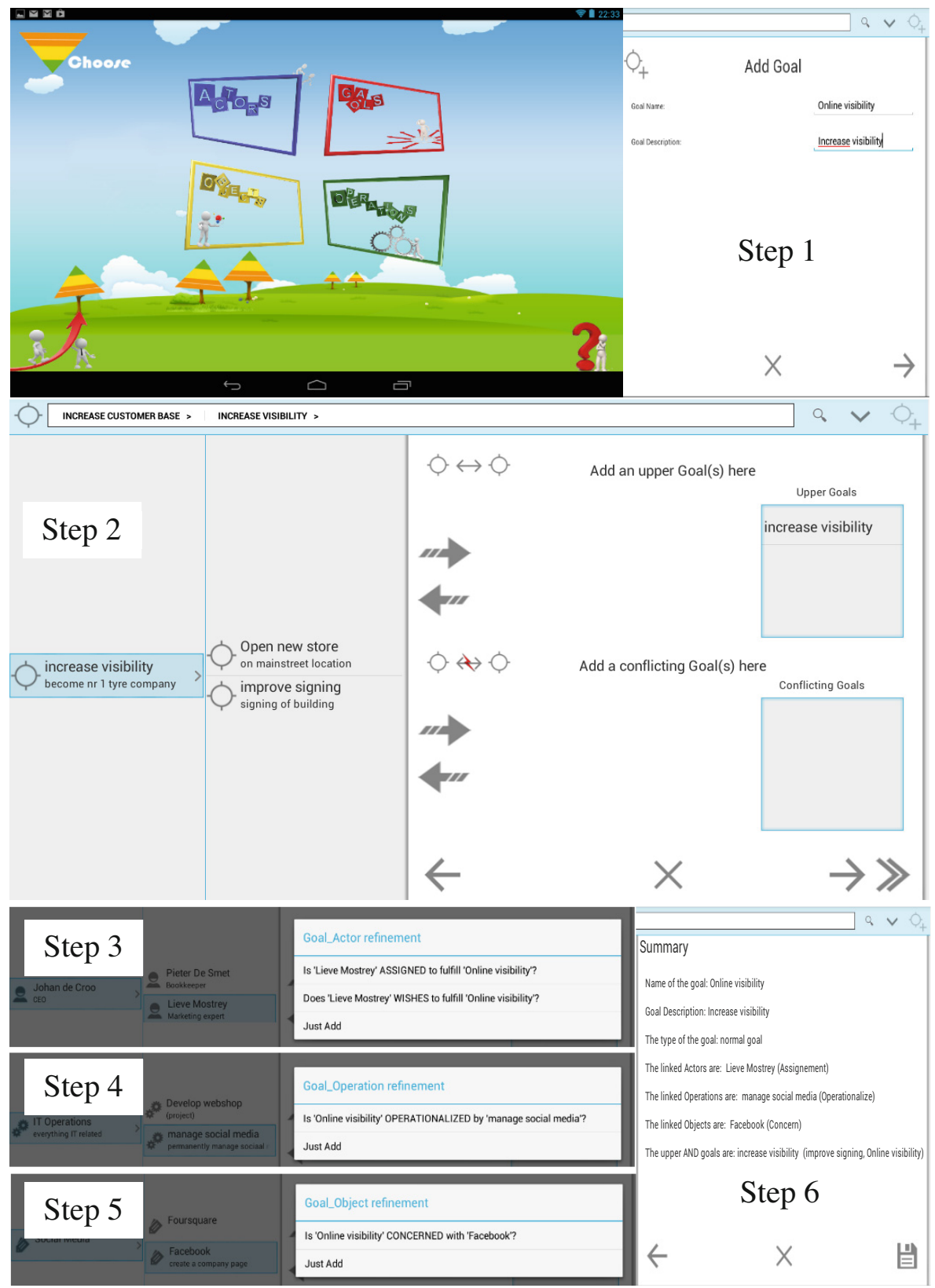

Fig. 6. Add goal 'online visibility' 


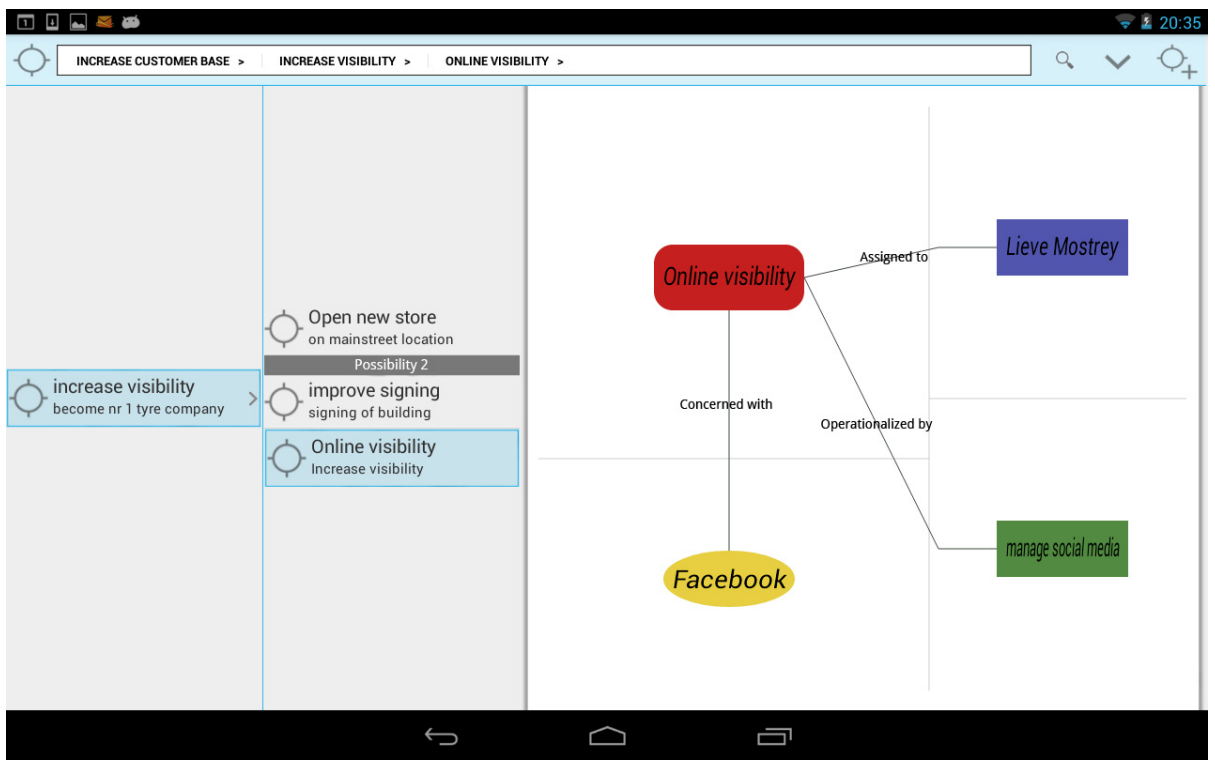

Fig. 7. Focused view 'online visibility'

\section{Conclusion}

EA approaches have been primarily developed for large companies, despite the importance of SMEs for economy. Tools furthermore have been developed to aid enterprise architects. In this paper, we investigated how tool support could be made usable for managers in SMEs. By using a goal-directed design approach in the tool development process, we came up with a radically different application. A tablet application has been proposed that is easy enough for managers and CEOs to use. First tests in SMEs have been very promising. They found it much easier to comprehend then traditional tools and were very enthusiastic about using a tablet. Further evaluation in practice will still be needed to evaluate the tool and the EA approach CHOOSE.

Although we focused on CEOs and managers working in SMEs, the goal-directed design approach could be expanded towards larger companies. Further research could find ways of better involving other stakeholders with easy to use applications. For example, employees could use a smartphone application to get an overview of their tasks and responsibilities, connected to the companies' goals. This could offer employees a sense of purpose.

\section{References}

1. Whittle, R., Myrick, C.: Enterprise Business Architecture: The Formal Link between Strategy and Results. IBM Systems Journal 1, 32-51 (2004)

2. Langenberg, K., Wegmann, A.: Enterprise architecture: What aspects is current research targeting. Laboratory of Systemic Modeling (2004) 
3. Ernst, A.M., et al.: Tool support for enterprise architecture management: strengths and weaknesses. In: 10th IEEE International EDOC, pp. 13-22. IEEE Press (2006)

4. European Commission: Are EU SMEs recovering from the crisis. Annual Report on EU Small and Medium sized Enterprises (2010)

5. Devos, J.: ICT-bestuur voor kleine en middelgrote ondernemingen (2011)

6. Bernaert, M., Poels, G.: The quest for know-how, know-why, know-what and know-who: using KAOS for enterprise modelling. In: Salinesi, C., Pastor, O. (eds.) CAiSE Workshops 2011. LNBIP, vol. 83, pp. 29-40. Springer, Heidelberg (2011)

7. Bernaert, M., et al.: Enterprise Architecture for Small and Medium-sized Enterprises: a Starting Point for Bringing EA to SMEs, Based on Adoption Models. In: Devos, J., Van Landeghem, H., et al. (eds.) Information Systems and Small and Medium-Sized Enterprises (SMEs): State of Art of IS Research in SMEs. Springer, Berlin (2013)

8. Schekkerman, J.: Enterprise Architecture Tool Selection Guide v5.0. Institute for Enterprise Architecture Developments (2009)

9. Kurpjuweit, S., Winter, R.: Viewpoint-based meta model engineering. In: EMISA Workshop, pp. 143-161 (2007)

10. IBM System architect, http://www-01.ibm.com/software/awdtools/systemarchitect/

11. Troux Metis, http://www.troux.com/products/troux_software/

12. Beck, T., Demirguc-Kunt, A.: Small and medium-size enterprises: Access to finance as a growth constraint. J. Bank Financ. 30(11), 2931-2943 (2006)

13. Cooper, A., et al.: About face 3: the essentials of interaction design. Wiley (2012)

14. Kamsties, E., et al.: Requirements engineering in small and medium enterprises. Requir. Eng. 3(2), 84-90 (1998)

15. Hankinson, A., et al.: The key factors in the small profiles of small-medium enterprise owner-managers that influence business performance: An international research project UK survey. Int. J. Entrep. Behav. Res. 3(3), 168-175 (1997)

16. Malhotra, R., Temponi, C.: Critical decisions for ERP integration: Small business issues. Int. J. Inform. Manage. 30(1), 28-37 (2010)

17. Colborne, G.: Simple and Usable Web, Mobile, and Interaction Design. New Riders (2010)

18. Shneiderman, B., Ben, S.: Designing the user interface. Pearson Education, India (1998)

19. Rising popularity of tablet use in small business community, http: / / www. forbes.com/sites/thesba/2012/04/03/ rising-popularity-of-tablet-use-in-small-business-community/

20. Davidson, M.J., et al.: Mental models and usability. Cognitive Psychol. 15 (1999)

21. Norman, D.A.: Affordance and design, http: / /www.liacs.nl/ fverbeek/courses/hci/ AffordancesandDesign.pdf

22. Norman, D.A.: The design of everyday things. Basic Books (2002)

23. IBM: Object-Oriented Interface: IBM Common User Access Guidelines. Design (1992)

24. Hertwig, R., Todd, P.M.: More is not always better: The benefits of cognitive limits (2003)

25. Thompson, D.V., et al.: Feature fatigue: When product capabilities become too much of a good thing. J. Marketing Res., 431-442 (2005)

26. Iyengar, S.S., Lepper, M.R.: Rethinking the value of choice: a cultural perspective on intrinsic motivation. J. Pers. Soc. Psychol. 76(3), 349 (1999) 